Wang Xiao-Dong (Orcid ID: 0000-0003-0574-4423)

Yoshifumi Futaana (Orcid ID: 0000-0002-7056-3517)

Galli Andre (Orcid ID: 0000-0003-2425-3793)

Wurz Peter (Orcid ID: 0000-0002-2603-1169)

\title{
Energy Spectral Properties of Hydrogen Energetic Neutral Atoms Emitted from the Dayside Atmosphere of Mars
}

X.-D. Wang ${ }^{1 *}$, S.Barabash ${ }^{1}$, Y. Futaana ${ }^{1}$, V. Shematovich ${ }^{2}$, A. Galli ${ }^{3}$, and P. Wurz

${ }^{1}$ Swedish Institute of Space Physics, Kiruna, Sweden

${ }^{2}$ Institute of Astronomy, Russian Academy of Sciences, Moscow, Russia

${ }^{3}$ Universität Bern, Bern, Switzerland

Corresponding author: Xiao-Dong Wang (wang@irf.se)

Key Points:

- We use two-segment power laws to fit the energy spectra of sunward-flowing hydrogen energetic neutral atoms (HENA) observed at Mars.

- Observed HENAs have higher fluxes and more high-energy component than the modeled backscattered HENAs.

- The more high-energy component implies additional sources of sunward HENAs, most likely the charge-exchange HENAs in the shocked solar wind.

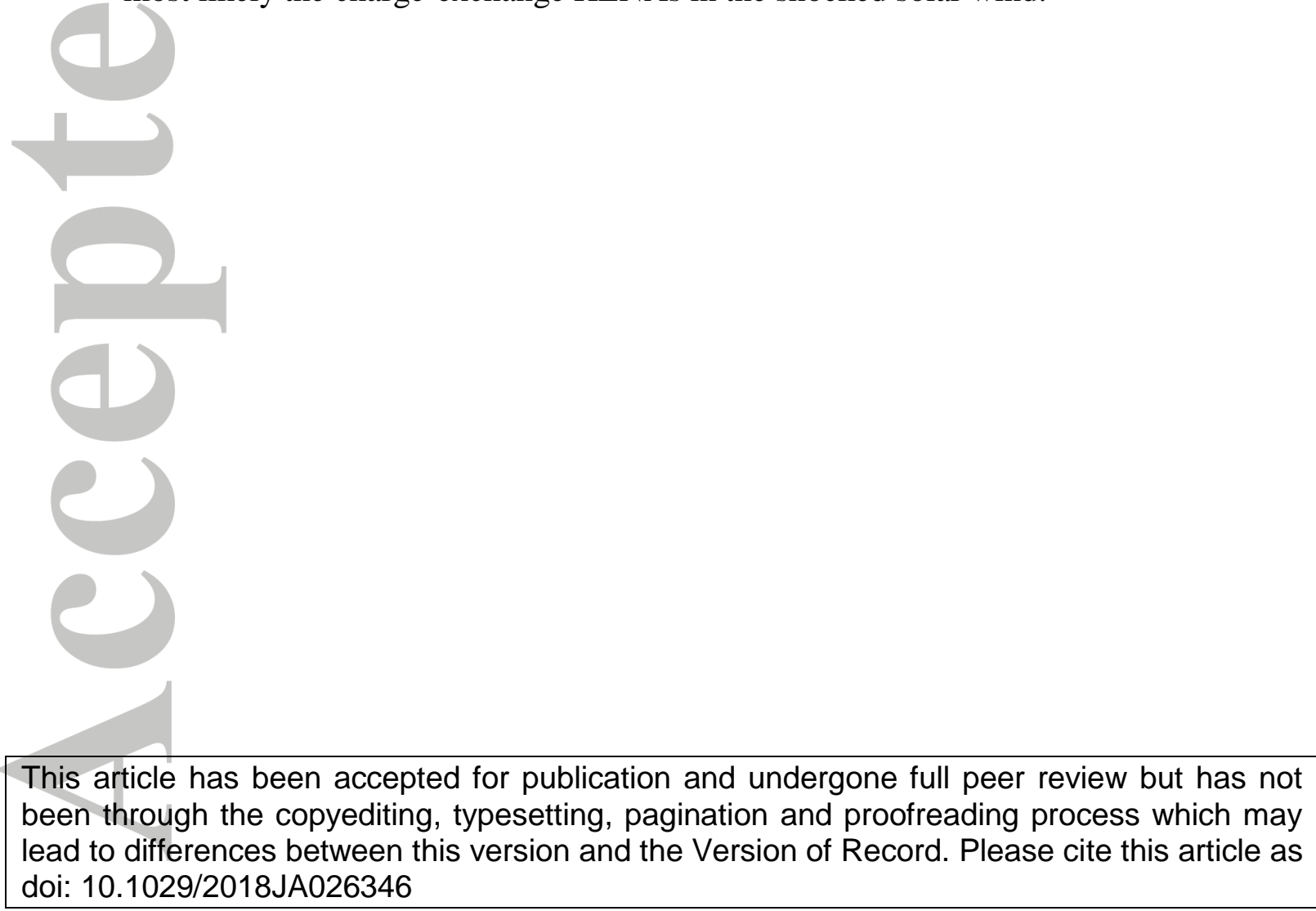

(C) 2019 American Geophysical Union. All rights reserved. 


\begin{abstract}
The energy spectrum of hydrogen energetic neutral atoms (HENA) emitted from the dayside atmosphere of Mars reveals the physical processes of their origin and provides means for remote-sensing on the Mars plasma environment. We first compile all available energy spectra of sun-ward flowing HENA using high energy resolution data from the Neutral Particle Detectors on Mars Express from February to June 2004. The median value of the HENA directional flux is $1.12 \times 10^{5} \mathrm{~cm}^{-2} \mathrm{~s}^{-1} \mathrm{sr}^{-1}$ from 0.3 to $5 \mathrm{keV}$, making Mars a bright source of HENAs. The energy spectra of the measured HENAs can be approximated by a two-segment power-law spectra. Then we compared the measured statistical energy spectra with those from a kinetic particle transport code. The comparison indicates that the measured HENA energy spectrum is harder (more high-energy components) than the modeled backscattered HENAs. We propose that a population of sunward-flowing, charge-exchanged HENAs produced in the dayside magnetosheath is the most probable reason for this difference. This HENA population with unexpected energy spectrum highlights the kinetic nature of the dayside magnetosheath of Mars.
\end{abstract}

\title{
Plain Language Summary
}

We study the measurements of fast hydrogen atoms moving towards the Sun from the dayside upper atmosphere of Mars. These measurements were done by the Neutral Particle Detectors on Mars Express spacecraft from February to June in 2004. We have got a curve showing how many $\mathrm{H}$ atoms move within a certain speed range. This so-called energy spectrum can tell us where these atoms come from and what has happened to them. Previous studies thought these $\mathrm{H}$ atoms are originally protons in the solar wind and are neutralized and scattered by the molecules high in Martian atmosphere. Our study, with the help of computer simulations, shows that the origin is correct, whereas scattering is not enough to explain the observed energy spectrum. We think that the $\mathrm{H}$ atoms we detected also contain those from solar wind protons that are deflected by the magnetic field around Mars. This study reveals more insight on how Mars atmosphere reacts to the supersonic solar wind and is related to the bigger question: how did Mars lose most of its atmosphere and water?

\section{Introduction}

Mars does not possess a global magnetic field (Acuna, 1998), and the solar wind can thus reach low altitudes before it is deviated by the induced magnetic boundary (IMB) located at about 0.2 Mars radii altitude at the sub-solar point (Dubinin et al., 2006). At this distance, the neutral atmospheric density is high enough (Chaufray et al., 2008, 2009) to cause frequent charge-exchanging collisions with the solar wind protons and produce significant fluxes of hydrogen energetic neutral atoms (HENA). Both the undisturbed and the shocked solar wind are partially neutralized by the exosphere and result in a total HENA flux that makes a few percent of the original solar wind proton flux (Kallio et al., 1997; Wang et al., 2018). These HENAs are called the charge-exchange (CX) HENAs. The CX HENAs are not affected by the electromagnetic field, therefore they can precipitate onto the upper atmosphere or fly into space in the tangents of the trajectories of their parent protons. The shocked solar wind protons sometimes also cross the induced magnetospheric boundary (IMB) and precipitate 
onto the atmosphere. The precipitating HENAs and protons can collide with the atmospheric constituents and may be scattered as HENAs. A secondary HENA source is the Martian hydrogen exosphere (Lich htenegger et al. firstly be ionized and then exchange charges with other exospheric atoms to produce planetary HENAs. The planetary HENAs have not been distinguished from the solar wind HENAs due to its low prodution rate, which is less than $\sim 1$ order of magnitude lower than that of the solar wind HENAs (Lichtenegger et al al., 2002; _Wang et_al._.2016). Therefore, we assume all detected HENAs to be solar wind origin herein.

Mars is a bright HENA source with substantial emissions of CX and scattered HENAs (Futaana et al., 2011). How to distinguish these two populations is still an unsettled issue in the application of planetary HENA diagnostics. In principle, one can separate the CX and the scattered populations by their different direction and energy distributions. The CX HENAs lose little energy in the CX collisions, therefore, they keep the original ion direction distribution, which is given by the solar wind proton temperature in this case. This feature is evident in the dayside HENA jets emitted from the subsolar region of Mars (Futaana et al., 2006b) and the tailward HENA flows in the tails of Mars and Venus (Galli et al., 2006; Galli, Fok, et al., 2008; Galli, Wurz, Bochsler, et al., 2008; Galli, Wurz, Kallio, et al., 2008). The scattered HENAs, on the other hand, are expected to show more isotropic emission. During a dayside passage, MEX observed a particle flux $(1.5-2) \times 10^{6} \mathrm{~cm}^{-2} \mathrm{~s}^{-1} \mathrm{sr}^{-1}$ between $0.2-2$ $\mathrm{keV}$, from the dayside atmosphere of Mars (Futaana et al., 2006a). Statistical studies also show that a moderate HENA flux can be observed in almost all directions at the dayside hemisphere (Wang et al., 2013).

The energy spectrum of HENAs is expected to provide more information on the parent ions of the HENAs and the plasma environment they pass through, for which very few results have been published. The energy spectra of the tailward HENA flows at Mars and Venus are consistent with that of the shocked solar wind at both planets (Galli, Fok, et al., 2008; Galli, Wurz, Kallio, et al., 2008), and those are the only HENA energy spectra available so far.

The observed energy spectrum of the sunward-moving HENAs has not been reported so far. It was long assumed that only HENAs scattered back by Martian upper atmosphere can move sunward. The properties of these backscattered HENAs have been modeled using various codes considering different physical processes. Kallio and Barabash (2000) analyzed the effects and fate of the precipitating HENAs produced from the upstream solar wind and indicated that a substantial fraction of the precipitating particle flux $(\sim 58 \%)$ and energy content $(\sim 33 \%)$ are scattered back, creating a HENA albedo of Mars. They also predicted a spectrum of the scattered HENAs originating from the precipitation of the neutralized, unshocked solar wind. Later, the high reflection reported by Kallio and Barabash (2000) was questioned. Shematovich et al. (2011) used a more sophisticated collision model and updated values for the cross section to study the transport of protons and energetic hydrogen atoms in the upper atmosphere, resulting in an updated the HENA albedo of 15\%--19\%. The spectral analysis on the sunward HENAs can provide evidence for the ENA albedo, if all sunward HENAs are indeed scattered.

On the other hand, latest simulation work predicted that some CX HENAs, in the absence of further collisions, can also move sunward (Wang et al. HENAs originate from sunward-gyrating protons in the dayside magnetosheath of Mars, 
which reflect the kinetic characteristics of the shocked solar wind plasma. The existence of this predicted population is yet to be investigated.

In this paper, we derive the statistical energy spectra of the sunward-moving HENAs from Mars Express observations and compare them with those of the CX HENAs and simulation results. This paper is organized as follows: in Section 2, we first introduce the Neutral Particle Detector (NPD), as a part of the ASPERA-3 instrument on board Mars Express, and then describe the observation geometry and the data processing. In Section 3, we first show a typical observation of the sunward HENAs, then show the statistical results of the spectral parameters from the whole dataset. In Section 4 and 5 we compare our current results with previous observations and models, respectively. Section 6 concludes the study.

\section{Instrumentation and Data Preparation}

\subsection{Instrumentation}

The NPD (Grigoriev, 2007) instrument is designed to measure the energetic neutral atoms with energies between $100 \mathrm{eV}$ and $10 \mathrm{keV}$. NPD is part of the ASPERA-3 instrument (Barabash et al., 2006), consisting of two identical sensors, NPD1 and NPD2. NPD is based on the time-of-flight (TOF) principle to measure the particle velocity. The incoming particles first pass an electrostatic deflector removing the charged component and a collimator to limit the elevation angle to $\sim 5^{\circ}$ width. Remaining neutral particles then pass through a pin-hole and impact on the START surface at a grazing angle of $15^{\circ}$. The secondary electrons from the collisions are collected to generate START signals, which are called START events. A fraction of the neutral particles continues to fly to the STOP surface, where STOP events are similarly generated. The pulse height $(\mathrm{PH})$ information of each STOP event is also recorded in high bit rate modes and can be used to roughly distinguish the particle mass (hydrogen or heavy) because for the same velocity more massive particles release, on average, more STOP electrons (Wurz et al., 2006). The distance between the START and the STOP surfaces is a constant and the time of flight of a particle is the time interval between a pair of coincident START and STOP events. Three STOP MCPs are installed along different azimuth angles to separate the arrival directions of the incoming particles. The angular response of each viewing sector to different incident directions is Gaussian-shaped in both elevation and azimuth directions. The full width of half maximum (FWHM) of the Gaussian response function in each direction is defined as its angular resolution, $\sim 5^{\circ}$ in elevation and $\sim 40^{\circ}$ in azimuth. The field of view (FOV) of one NPD sensor is $5^{\circ} \times \sim 90^{\circ}$ because the response functions overlap between neighboring sectors. NPD1 and NPD2 are installed such that their FOV planes are $\pm 15^{\circ}$ off the plane perpendicular to the spacecraft velocity, therefore they "scan" over the space below the spacecraft. More details of NPD functions are described in Grigoriev (2007).

The NPD operates in three modes: RAW, TOF and BIN mode. In the RAW mode, the instrument records the first 512 STOP events every second. The recorded information of each event includes the TOF with a resolution of $1 \mathrm{~ns}$ over the TOF window ( 0 to $2048 \mathrm{~ns}$ ), the pulse height of the STOP event, the incident direction, and the coincidence flag with a START event. The actual accumulation time $\Delta t$ to fill the 512 events usually is less than $1 \mathrm{sec}$ because the total coincident count rate $R_{\text {coin }}$ is almost always higher than 512/s. The TOF spectrum (the frequency of coincident events as a function of TOF) is the histogram of the coincident events in the unit of $\mathrm{s}^{-1} \mathrm{~ns}^{-1}: C R_{T}=n_{v a l, T O F} / \Delta t / \mathrm{d} T$, where $n_{v a l, T O F}$ is the number of 
valid events within the TOF bin of width $\mathrm{d} T$ (=1 ns in this case) at a specific TOF value $T$. In the TOF mode, the instrument TOF window is up to $2048 \mathrm{~ns}$ divided into 8-ns bins, inherently obtaining the TOF spectrum twice a second. No pulse height information is recorded in the TOF mode. The TOF information in the BIN mode is sparse; only 16 TOF bins are available. Thus, we investigate the dayside HENA emission from Mars with RAW and TOF modes in this study.

\subsection{Observation Geometry}

To investigate the HENA emitted from the dayside of Mars, at least one sector of NPD must cover the dayside atmosphere. This constraint limits the dataset to the period from 9 February to 20 June 2004. During this period, the typical observation geometry of NPD was similar to what is shown in Figure 1a in the spacecraft coordinate system. The spacecraft was on the dayside of Mars when the NPD began to operate in each orbit and then moved poleward. During the operation of NPD, the spacecraft kept its attitude in the Mars Solar Orbit (MSO) frame, so the viewing directions of NPD sectors also kept invariant, "panning" with the movement of the spacecraft on the trajectory. This observation geometry allows the NPD to sweep the dayside atmosphere, the region of our interest. The observation geometry was kept similar in all the data we select, although not exactly identical.
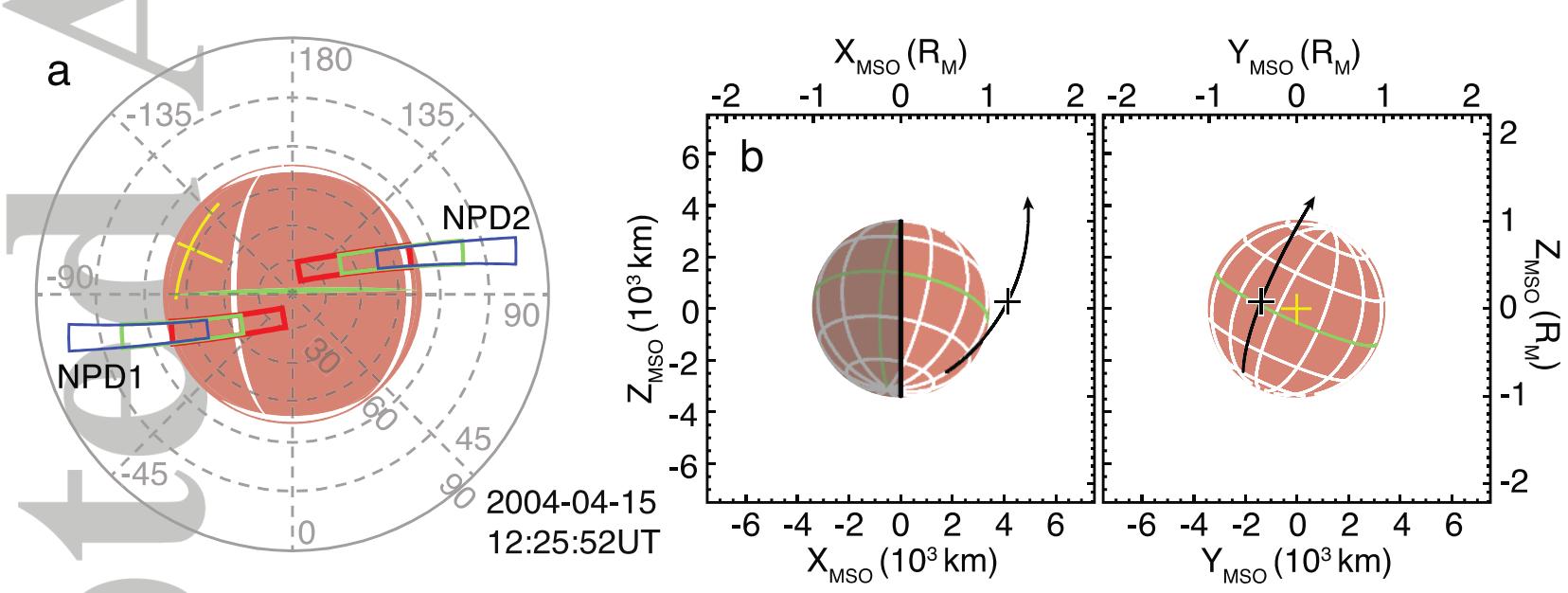

Figure 1: The typical observation scenario for sunward HENAs from Mars. The observations are from 12:12 UT to 12:51 UT on 15 April, 2004. Panels: (a) the viewing directions of NPD1 and NPD2 in the spacecraft frame at 12:25:52 UT. The grid in the instrument related frame shows the polar angle and the azimuth angle with intervals of $15^{\circ}$ and $45^{\circ}$, respectively. Red (Dir0), green (Dir1) and blue (Dir2) boxes illustrate the field of view of both NPD sensors. The solid sphere filled by brown color is the Mars disk seen from the current position. The yellow "+" marks the subsolar point on the Mars surface. The green and white curves on Mars surface are the equator and grids of the Martian aerographic frame, respectively. (b) The black curve with an arrowhead shows the trajectory of the spacecraft during the period of observation in the Mars Solar Orbit (MSO) frame. The "+" is the spacecraft position at 12:25:52 UT when the view in Panel a was obtained.

Figure 1 shows the NPD observation centered at 12:25:52 UT, 15 April 2004, when the spacecraft pointing and position was optimal for detecting sunward HENAs. On this orbit the NPD operated from 12:12 UT to 12:51 UT, as the spacecraft moved from the southern to the northern hemisphere. The spacecraft outbound trajectory crossed the IMB at 12:20:22 UT 
and the bow shock at 12:34:52 UT. At the time shown in Figure 1a, the spacecraft was in the dayside magnetosheath. The viewing directions of the NPD2's three angular sectors are tailward-facing, meaning that NPD2, unlike NPD1, should be free of tailward chargeexchange HENAs and the protons neutralized inside the instrument. The NPD2 directions Dir0 and Dir1 (red and green boxes in Figure 1) were looking down at the upper atmosphere, covering solar zenith angles from $20^{\circ}$ to $50^{\circ}$ and thus detecting sunward-flowing HENAs.

\section{NPD data processing and statistical characteristics}

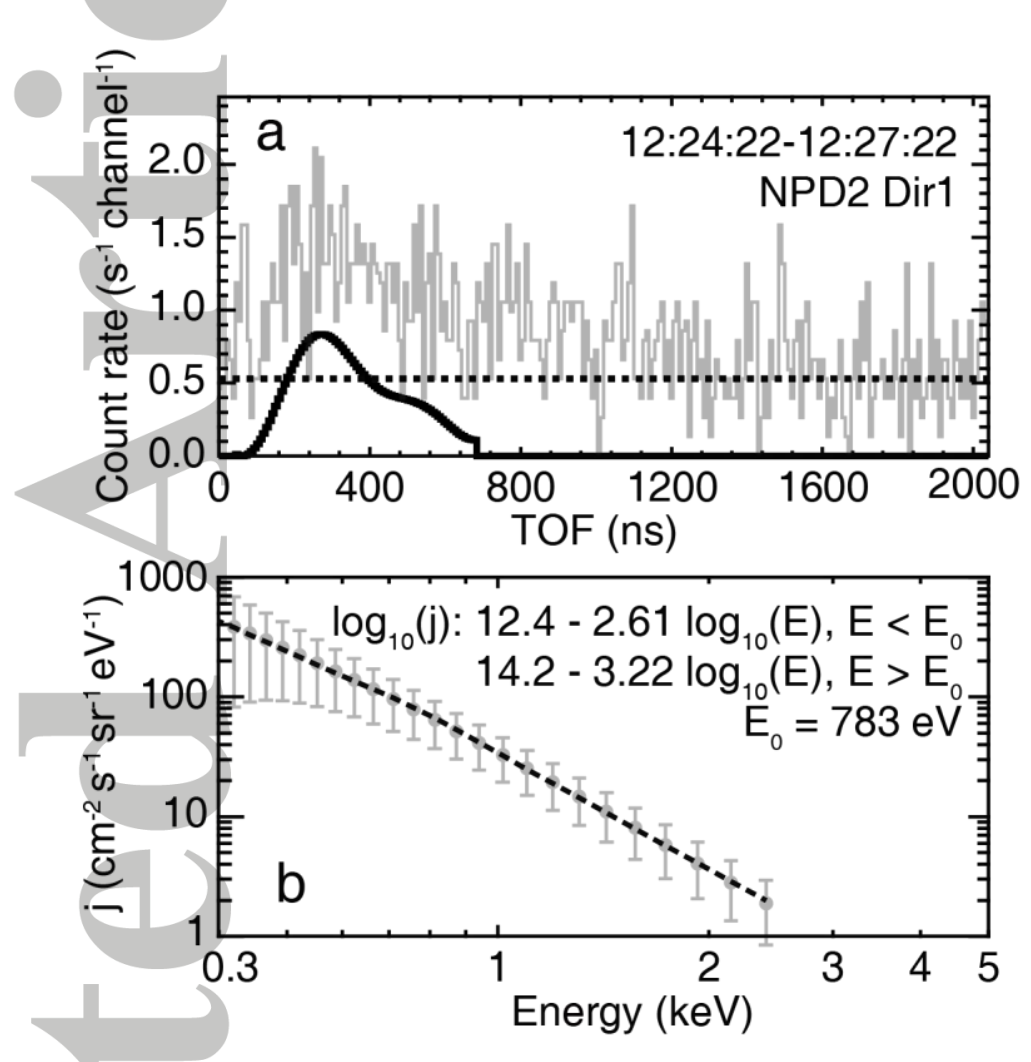

Figure 2: (a) An observed TOF spectrum during the period shown in Figure 1. The thin gray curve and the thick black curve show the averaged and background-subtracted TOF spectrum, respectively. The dotted line is the average background level estimated from the averaged TOF spectrum. (b) The energy spectrum converted from the reconstructed TOF spectrum in panel a (gray dots with error bars). The dashed black line shows the fitted results of the two-segment power law with a fitting equation also shown.

The data processing procedure to obtain the TOF spectra includes two steps. First, we average the TOF spectrum over time, since the counting statistics of a TOF spectrum obtained in a single observation is usually low. Here we take 3-minute average (i.e., 180 raw TOF spectra). The 3-minute accumulation time is long enough to obtain sufficient statistics, while is still keeping similar observation geometries. Figure 2 a shows the average TOF spectrum observed during the period 12:24:22 UT--12:27:22 UT (thin gray curve). By following the same procedure as described in Galli et al. (2006), we get the reconstructed TOF spectrum as shown by a thick curve in Figure 2a. In this period, the reconstructed TOF spectrum shows a broad peak at $230 \mathrm{~ns}$, which is consistent with the previous report of backscattered HENA observations (Futaana et al., 2006a). 
We convert the reconstructed TOF spectrum to the differential flux intensity as function of energy by applying the following formula:

$$
j_{i}=\frac{C_{i}}{G_{0} \cdot \varepsilon\left(E_{i}\right) \cdot \Delta E_{i}}
$$

where the subscript $i$ refers to the $i$-th TOF channel, $j_{i}$ is the differential flux intensity in the unit of $\mathrm{cm}^{-2} \mathrm{~s}^{-1} \mathrm{sr}^{-1} \mathrm{eV}^{-1}, C_{i}$ is the count rate in channel $i, G_{0}$ is the pure geometric factor for hydrogen ENAs independent of energy, $\varepsilon\left(E_{i}\right)$ is the detection efficiency at the energy $E_{i}$, and $\Delta E_{i}$ is the energy bandwidth of channel $i$. Here we assumed all the particles are hydrogen atoms. The uncertainty of the converted energy spectrum are mainly contributed by the counting statistics, the estimation of UV background, and the detection efficiency for atoms at energies below $0.3 \mathrm{keV}$ (Galli et al., 2006).

To describe the spectral shape, we follow the approach developed by Galli et al., (2006) and fit the spectrum with a two-segment power law function.

$$
j(E)= \begin{cases}a_{1} E^{k_{1}}, & E<E_{0} \\ a_{2} E^{k_{2}}, & E \geq E_{0}\end{cases}
$$

where $k_{1}$ and $k_{2}$ are the slopes of the spectrum in the low- and high-energy part, respectively, $E_{0}$ is the transition energy between the two branches. The fitting is done on the logarithms of the differential flux in the spectrum with the least-square approach. In the fitting of an energy spectrum, we discard any channel with an uncertainty larger than the differential flux intensity value. In the case of the example shown in Figure $2 b$, we obtained a transition energy of $E_{0}=783 \mathrm{eV}$, and the two power-law indices $k_{1}=-2.61$ and $k_{2}=-3.22$. We also calculate the directional flux $f$ (in the unit of $\mathrm{cm}^{-2} \mathrm{~s}^{-1} \mathrm{sr}^{-1}$ ) by integrating $j$ over the $0.3 \mathrm{keV}--5$ keV range.

We analyze 96 TOF spectra with ENA contributions significantly above the background from the available dataset and perform the processing described above. Statistics of the spectral features, namely the histograms of $E_{0}, k_{1}, k_{2}$, and $f$, are shown in Figure 3 . The medians of the slopes $k_{1}$ and $k_{2}$ are -2.74 and -3.13, respectively, and the median of the transition energy $E_{0}$ is $607 \mathrm{eV}$. However, the transition energy is not so well-constrained because the slopes of the two segments are close, which may explain the rather broad distribution of $E_{0}$. The integral flux over the energy of $0.3 \mathrm{keV}--5 \mathrm{keV}$ is $f=(0.6-2) \times 10^{5} \mathrm{~cm}^{-2} \mathrm{~s}^{-1} \mathrm{sr}^{-1}$ around its median value of $f_{\text {int }}=1.12 \times 10^{5} \mathrm{~cm}^{-2} \mathrm{~s}^{-1} \mathrm{sr}^{-1}$.
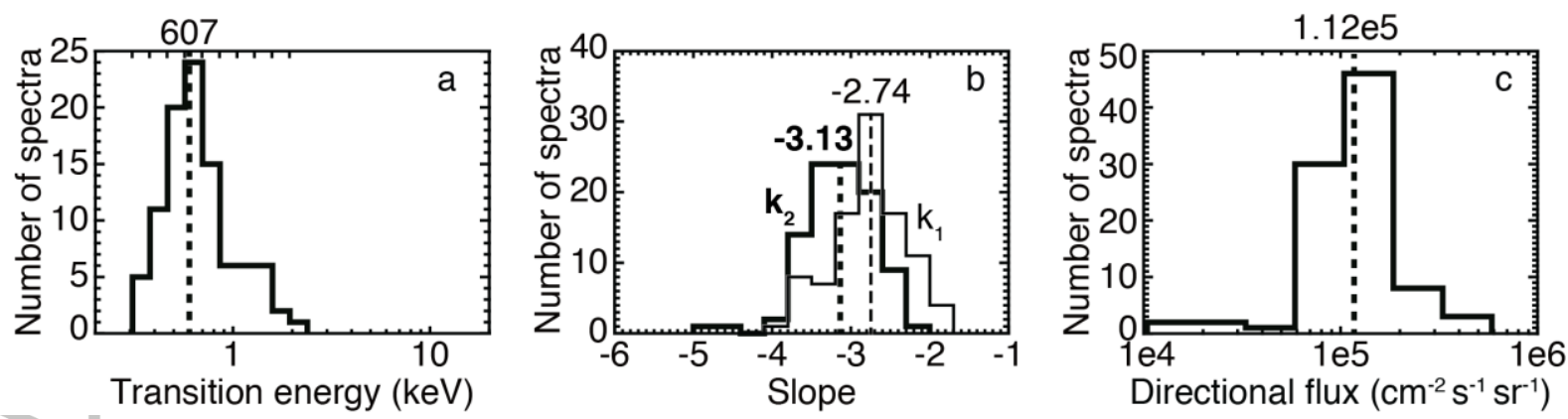

Figure 3: Histograms of fitted spectral parameters (Eq. 2) of the measured HENAs and the median values (dashed lines and the numbers). (a) The transition energy $E_{0}$; (b) The low- 
energy segment slope $k_{1}$ and the high-energy segment slope $k_{2}$; (c) The directional fluxes obtained by integrating the differential flux over the energy range of $0.3 \mathrm{keV}$ to $5 \mathrm{keV}$.

\section{Comparison with previous observations at Mars and Venus}

As introduced in Section 1, almost all measured sunward HENAs originate from the solar wind since the production rate for the planetary HENAs is much lower ((Lichtenenegger et $\underline{\text { al }}$..2 2002) Wang et al., 2016). These sunward HENAs are produced as the solar wind protons approach and propagate through the Martian space environment. In principle, the properties of these sunward HENAs reflect the properties of the environment they pass through, namely, the neutral exosphere, the magnetosheath, and less frequently, the induced magnetosphere.

However, it is not feasible to derive the above information reliably by inverting the current HENA measurements. The reasons are: the non-unique nature of inversion, the highly fluctuating upstream solar wind conditions, the highly variable solar radiation level and therefore the exospheric density profile, and the uncertainty of the instrument. Therefore, we first compare our measurements with other HENA measurements with the same instrument to constrain at least the last factor. This comparison aims at identifying the relative differences between the properties of HENAs observed in different environments, and a qualitative explanation for these differences.

Table 1: Spectral parameters and integrated directional flux of different HENA populations wherever applicable. In this study, $k_{1}, k_{2}, E_{0}$, and $f_{\text {int }}$ are medians. The ranges are the standard deviation of individually fitted parameters. Values from other studies follow their respective references. $\mathrm{BS}=$ backscattering. $\mathrm{CX}=$ charge exchange.

\begin{tabular}{|c|c|c|c|c|c|c|}
\hline Population & $\begin{array}{c}f_{\text {int }}\left(10^{5} \mathrm{~cm}^{-2}\right. \\
\left.\mathrm{s}^{-1} \mathrm{sr}^{-1}\right)\end{array}$ & $\begin{array}{c}\text { Energy } \\
\text { range }(\mathrm{keV})\end{array}$ & $E_{0}(\mathrm{keV})$ & $k_{1}$ & $k_{2}$ & Source process \\
\hline $\begin{array}{c}\text { Mars sunward (this } \\
\text { study) }\end{array}$ & 1.12 & $0.3-5$ & $0.6 \pm 0.3$ & $-2.74 \pm 0.5$ & $-3.14 \pm 0.5$ & $\begin{array}{c}\mathrm{BS}+\mathrm{CX} \\
\text { (conclusion of this study) }\end{array}$ \\
\hline Mars atmospheric ${ }^{\mathrm{a}}$ & $7.5-10$ & $0.2-2.3$ & 1.1 & - & - & BS \\
\hline Mars tailward ${ }^{b}$ & $0.1-1$ & $0.3-5$ & 1.0 & -2.2 & -2.7 & $\mathrm{CX}$ \\
\hline Venus tailward $^{\mathrm{c}}$ & 1.2 & $0.2-10$ & $1.0 \pm 0.1$ & $-1.8 \pm 0.2$ & $-3.3 \pm 0.1$ & $\mathrm{CX}$ \\
\hline
\end{tabular}

Table 1 lists all the HENAs observed near Mars and Venus, most relevant to the sunward HENAs in this study. Futaana et al. (2006a) reported for a single observation that MEX observed a HENA flux of $(0.75--1) \times 10^{6} \mathrm{~cm}^{-2} \mathrm{~s}^{-1} \mathrm{sr}^{-1}$ between $0.2--2.3 \mathrm{keV}$ coming from the dayside atmosphere, most likely to be the scattered population. This flux value is about ten times higher than the average flux in our study, $1.12 \times 10^{5} \mathrm{~cm}^{-2} \mathrm{~s}^{-1} \mathrm{sr}^{-1}$. This discrepancy is likely due to Mars being in a disturbed space environment at the time of observations, and the resulting HENA flux in this case was thus higher than the average. Indeed, the peak energy of the scattered HENAs is $\sim 1.1 \mathrm{keV}$ in this event, which is even higher than the usual peak energy of the upstream solar wind. This unusually high peak energy indicates that the speed of the precipitating solar wind HENAs must have been even higher, consistent with a highly disturbed space condition. Futaana et al. (2006) proposed several explanations for such an intense event: higher upstream solar wind flux, lower IMB altitude, and/or precipitating protons backscattered as HENAs. In general, although the observation reported by Futaana et al. (2006) is the most similar measurement of HENAs to our current study, it can hardly 
verify our measurements or provide constraints on the underlying physics. Nevertheless, this is the only direct observation of sunward HENA emission from the dayside hemisphere of Mars.

Another HENA population of solar wind origin in a planetary environment is the tailward HENA flux at Mars and Venus (Galli, Fok, et al., 2008; Galli, Wurz, Kallio, et al., 2008). They are produced by the CX between the shocked solar wind protons and the planetary exosphere at the flank of the magnetopause. The tailward HENAs, therefore, have spectral characteristics different from that of sunward HENAs, due to the different source plasma populations and the production mechanisms. In previous works, the spectra of tailward HENAs were also fitted with Equation 2 determined by the four parameters: the transition energy $E_{0}$, the differential flux $j_{0}$ at $E_{0}$, and the two slopes $k_{1}$ and $k_{2}$. The differential flux $j_{0}$ in our current study is higher than that of the tailward HENAs at Mars while comparable to that at Venus. The transition energy $E_{0}$ for Mars sunward HENAs is $0.6 \pm 0.3 \mathrm{keV}$, lower than that of the tailward HENAs at Mars $(1 \mathrm{keV})$ and Venus $(1.1 \pm 0.1 \mathrm{keV})$. Moreover, the spectrum of the sunward HENAs appears to be softer than that of the CX HENAs in the lowenergy part, while having the same slope in the high-energy part (within the current measurement uncertainty).

The flux of sunward HENAs lies between that of the tailward populations at Mars and Venus. This result is realistic despite that the Mars sunward HENAs should have undergone more collisions than that of the tailward HENAs. The exospheric column density along a proton trajectory is higher in the subsolar region than that at the flank magnetopause, meaning that the HENA flux precipitating to the subsolar exobase exceeds the tailward emissions.

Therefore, the backscattered HENA flux could be higher than the tailward flux at Mars, or comparable to the tailward flux at Venus even though the solar wind proton flux at Venus is about four times that at Mars. However, the comparison of fluxes gives limited information on the physical processes the sunward HENAs have experienced. Other spectral parameters may provide more insight.

Both the lower transition energy and the softer spectra of the sunward-flowing ENAs suggest that they indeed contain a backscattered population. The backscatter of a particle requires large $\left(>90^{\circ}\right)$ deviation from its original velocity direction, which can usually be satisfied only after tens to hundreds of collisions due to the tiny differential cross section for backscattering. The accumulated energy loss in total can reach a few hundreds of eV (Kallio \& Barabash, 2000). As a result, the spectrum of backscattered HENAs is shifted towards lower energies. By contrast, the CX HENAs lose negligible energy in the charge exchange interactions when they are produced. More collisions will also cause a softer energy spectrum than the incident population by increasing the low-energy HENA fluxes. An observational example is the scattering of solar wind protons by the porous lunar regolith, where the spectrum of the scattered HENAs showed a much softer energy distribution below the solar wind peak energy (Futaana et al., 2012).

To summarize, the comparisons with previous observations suggest that the observed flux and spectra of sunward HENAs are consistent with the contribution of a backscattered population. However, it is challenging to draw more constraints regarding the physical processes producing the sunward HENAs as well as the relevant space plasma environment of Mars: the scatter rate, the properties of the source populations of the sunward HENAs. One needs to know the upstream solar wind conditions and the exospheric density distribution to constrain the highly variable plasma environment with the limited data coverage. Therefore 
we need to compare current observations with numerical simulations, where all the information is defined.

\section{Comparison with models}

\subsection{Comparison with Published Model Results}

The comparisons with previous observations suggest the sunward HENAs contain the scattered population, but could not quantify its properties. Therefore we compare our observations with models assuming them to be all scattered population. We first compare with published model results to check if those investigated processes can explain our observation.

Previous models predicted the scatter rate of the upstream solar wind HENAs on the upper atmosphere, ranging from 15\% (Shematovich et al., 2011) to 58\% (Kallio \& Barabash, 2000), using different models and assumptions. Moreover, Shematovich et al. (2011) also simulated the spectrum of scattered HENAs from the precipitation of the upstream solar wind HENAs. If we assume all measured sunward HENAs are scattered solar wind HENAs, and the scattering is isotropic, our measured flux of $1.12 \times 10^{5} \mathrm{~cm}^{-2} \mathrm{~s}^{-1} \mathrm{sr}^{-1}$ corresponds to a HENA precipitation flux from $1.2 \times 10^{6} \mathrm{~cm}^{-2} \mathrm{~s}^{-1}$ (scatter rate $58 \%$ ) to $4.7 \times 10^{6} \mathrm{~cm}^{-2} \mathrm{~s}^{-1}$ (scatter rate $15 \%)$. There has been no direct measurement of the precipitating HENAs at Mars. The indirect measurement of the re-ionized precipitating HENAs shows that $\sim 0.5 \%$ of the upstream solar wind protons are neutralized by the bow shock altitude (Halekas, 2017, Figure $3)$. Since their observations were done in a similar season and solar activity level of our observations, we can use this value as the reference precipitating ratio for the upstream solar wind HENAs. The typical upstream solar wind proton flux at Mars ranges from $3 \times 10^{7} \mathrm{~cm}^{-2} \mathrm{~s}^{-1}$ to $9 \times 10^{7} \mathrm{~cm}^{-2} \mathrm{~s}^{-1}$ (cf. Ramstad et al., 2015, Figure 3), and there is no observational evidence of significant deviation from this range during the interval of our observations. A $\sim 0.5 \%$ precipitation fraction of upstream solar wind would thus produce a flux of $(1.5--4.5) \times 10^{5} \mathrm{~cm}^{-}$ ${ }^{2} \mathrm{~s}^{-1}$ for the precipitating HENAs.

This precipitation flux is about one order of magnitude lower than what is needed to produce a scattered HENA flux comparable to what we measure. A factor of 3 uncertainty is estimated to be contributed by the fluctuations of the upstream solar proton flux, the column density of the exosphere, and the statistical scattering of measured HENA flux. Therefore, at least a factor of 3 difference exists between the measured sunward HENA flux and the expected flux of scattered upstream solar wind HENAs.

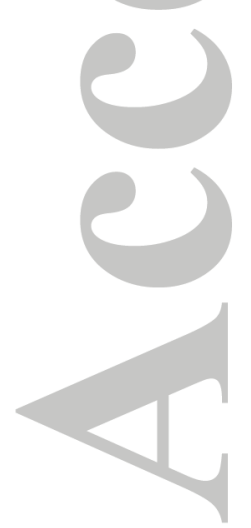




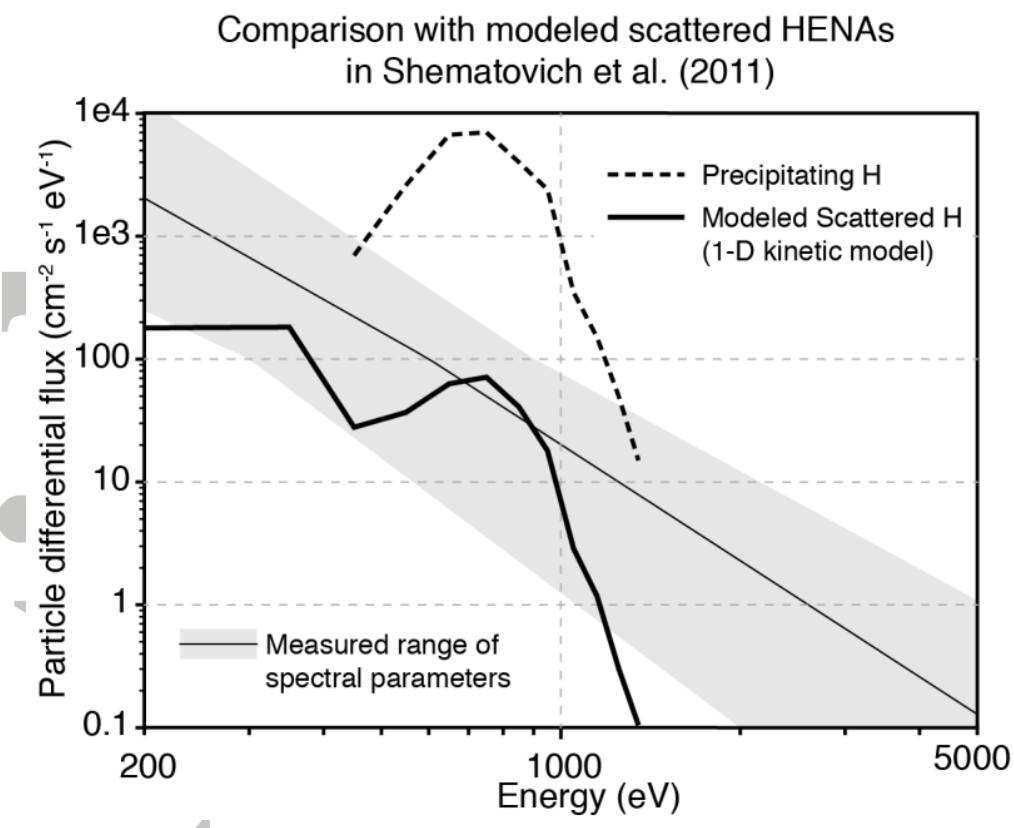

Figure 4: Comparison between the measurements and results of Shematovich et al. (2011).

The gray shaded area shows the parameter range derived from the observations in our current study (Table 1). The gray shaded area does not include the observational uncertainty in Figure $2 \mathrm{~b}$. The dashed curve is the energy spectrum of precipitating HENA as the input to the kinetic code. The thick solid curve shows the energy spectrum of upward hydrogen atoms simulated by the kinetic code. HENA = hydrogen energetic neutral atom.

The energy spectrum of the scattered HENAs has also been investigated with models. Shematovich et al. (2011) investigated transport processes for the solar wind HENAs produced upstream of the bow shock. They predict that at least $15 \%$ of precipitated solar wind HENAs are backscattered as sunward HENAs. The scattered HENA energy spectrum contains more low-energy component than the solar wind HENAs. Figure 4 shows the comparison between modeled predictions and our observation in this study. Instead of the individual measured spectrum, we visualize the parameter range of our measurements in this study to emphasize the statistical nature of this comparison. The comparison shows that the simulation in Shematovich et al. (2011) did not predict as much upward H fluxes above and below the transition energy $E_{0}$. The measured spectra show less difference between the slopes above $E_{0}$. Below $E_{0}$ the measurement uncertainty grows rapidly with decreasing energy. Therefore, we cannot draw a definite conclusion on the comparison.

To summarize, compared to published model results, our measurements show an integral flux that is $\sim 3$ times the expectated values, with a spectrum that contains more high-energy components. These discrepancies shall be explored by new simulations considering HENA sources in addition to the solar wind HENA precipitation.

\subsection{Evaluation of Additional HENA Sources}

Two additional source populations could potentially contribute to the difference between the modeling and observations: the precipitating protons and the magnetosheath CX HENAs. The latter could either be scattered or move in sunward directions. 
The proton precipitation is the less likely due to its low occurrence rate. Proton precipitation refers to the protons of solar wind origin observed below the IMB (Diéval et al., 2012). There, the precipitating protons will collide with exospheric atoms and molecules and are scattered back as neutrals. The precipitating protons have harder spectra than the upstream HENAs (Diéval et al., 2013). Therefore they also produce scattered HENAs with harder spectra, which qualitatively agree with the observed spectra in the high-energy part (energies higher than the transition energy $E_{0}$ ). However, proton precipitation is a rare phenomenon at Mars since it was observed only $3 \%$ of the time with the suitable observation configuration (Diéval et al., 2013), and no evidence shows a higher occurrence rate during the NPD observation period. Weighted by this low occurrence rate, the proton precipitation is unlikely to be the main reason for the harder spectrum of the observed HENAs compared to the kinetic transport model.

The magnetosheath HENAs are produced by the CX reaction between the shocked solar wind protons and the exospheric atoms (mostly atomic hydrogen) in the dayside magnetosheath. The magnetosheath HENAs keep the velocity distribution of the shocked solar wind protons in the dayside magnetosheath, which may cause two situations:

1. Most of the magnetosheath HENAs have tailward velocity components. Therefore they continue to cross the horizontal magnetic field of the dayside magnetosphere after birth and eventually precipitate to the exobase. There precipitating HENAs can be scattered in a similar process to that of the upstream solar wind HENAs and contribute to the measured sunward HENA flux.

2. The shocked solar wind protons have a high temperature. In the subsolar region, they are even subsonic. The individual shocked solar wind protons could gyrate, and thus the resultant HENAs may have sunward velocity components in the subsolar magnetosheath.

Previously published simulations of HENAs from planets did not consider the magnetosheath populations. Therefore we employ new simulations to investigate their effects.

We model the production of magnetosheath CX HENAs with a 3-D quasi-neutral hybrid code combined with a spherical exosphere model of Mars (Kallio et al., 2010; Wang et al., 2018). We adopt the results of the nominal slow solar wind run in Wang et al., (2018), which employs a proton density of $2.7 \mathrm{~cm}^{-3}$, a velocity of $335 \mathrm{~km} / \mathrm{s}$, and an exosphere containing both cold and hot populations for the atomic hydrogen and oxygen under low solar radiation conditions. We calculate the energy spectra of the precipitating and sunward-moving HENAs including both the upstream and the magnetosheath populations (Figure 5, dashed curve: precipitation, blue solid curve, sunward-moving). As a validation, the simulated energy spectrum of magnetosheath HENAs agrees well with the measured energy spectrum of magnetosheath protons (Nilsson et al., 2012).

Then we feed the precipitating spectrum to the 1-D kinetic code and model the spectrum of the scattered HENAs (Figure 5, gray dots). The setup of the 1-D kinetic model is exactly the same as in Shematovich et al. (2011). The resulting spectrum of scattered HENAs is also fitted with the two-segment power law function (Figure 5, thick black line, Equation 2). 


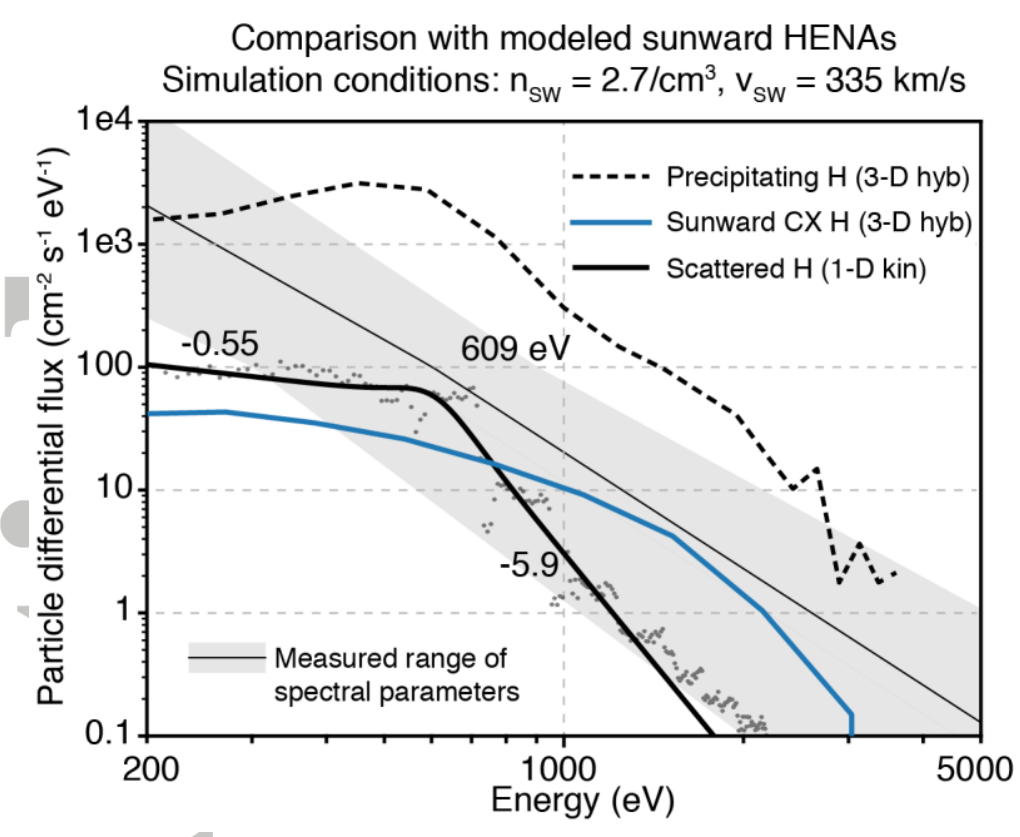

Figure 5: Comparison between the measurements and the modeled HENA spectra in this study. The measurement (the gray shade) is identical to that in Figure 4. The dashed line and the blue line show the spectra of the precipitating and the sunward-moving charge-exchange (CX) HENAs, respectively, including both the upstream solar wind and the magnetosheath productions. These spectra are modeled by a 3-D hybrid code (Wang et al., 2016, 2018). The scattering of the precipitating HENAs is modeled by the same 1-D kinetic transport code in Shematovich et al. (2011). We fit the resulting spectrum (gray dots) with the two-segment power law function (Equation 2, thick black curve) labeled with the transition energy $E_{0}(609$ $\mathrm{eV}$ ) and two slopes ( -0.55 below $E_{0}$ and -5.9 above $E_{0}$ ). hyb = hybrid model (Kallio et al., 2010; Wang et al., 2016, 2018), kin = kinetic model (Shematovich et al., 1994, 2011)

The kinetic model correctly reproduces the spectral transition energy of the backscattered HENAs, even without the magnetosheath HENA precipitation. Additionally, it shows that after the inclusion of magnetosheath HENA precipitation, the scattered HENAs indeed show more high- and low-energy components than that from the upstream HENA precipitation only. Both slopes $\left(k_{1}=-0.55, k_{2}=-5.9\right)$ in our current simulation are getting closer to the observed ranges. This trend is consistent with our hypothesis 1 , that the observed sunward HENAs indeed include the backscattered magnetosheath HENAs.

However, the simulated scattered HENAs has a softer spectrum above the transition energy $\left(k_{2}=-5.9\right)$ than the measured HENAs, indicating that including the magnetosheath precipitation in this simulation is not enough to explain the spectrum of the sunward HENAs above the transition energy. This discrepancy is significant, given that the measured HENA flux in this energy range of the spectrum has relatively small observational uncertainties (see Figure 2b).

Our simulations nicely reproduce the sunward CX HENAs contribution to the measured HENA spectrum, supporting our hypothesis 2. The sunward CX HENAs were first reported by Wang et al. (2016) in a simulation to investigate the HENA emission from the dayside hemisphere of Mars. In their simulation results, a flux of $5 \times 10^{4}--10^{5} \mathrm{~cm}^{-2} \mathrm{~s}^{-1} \mathrm{sr}^{-1}$ in the sunward direction was observed from the subsolar region with $<30^{\circ}$ solar zenith angle (Wang 
et al., 2016, Figure 5 and Figure 8). The spectrum of this population, as demonstrated in this study, fits well the observed spectrum of sunward HENAs above the transition energy.

The dayside magnetosheath is full of perturbations and waves excited by the interaction of the solar wind with the IMB. The proton energy distribution in the magnetosheath is thus much broader than that in the solar wind, while the bulk flow velocity is significantly reduced, especially in the vicinity of the subsolar point. There, proton thermal speed might exceed their bulk flow speed, which means that during half of their gyrations, magnetosheath ions will have a sunward velocity component. During that phase, if they exchange charge with the background neutral exosphere particles, they will become sunward HENAs. Because MEX was upstream of the bow shock during the observations used in this study, NPD could not distinguish if the HENAs are produced in the magnetosheath or below the IMB as long as they are in the FOV. Therefore, HENAs produced by CX in the magnetosheath but with a sunward velocity component are received and contribute to the high-energy part of the spectrum.

To summarize, our observations of sunward HENAs indicate the existence of sunwardgyrating protons in the dayside magnetosheath of Mars, demonstrating the kinetic nature of Martian dayside magnetosheath.

\section{Conclusions}

We have reported the statistical properties of the energy spectrum of the sunward-moving HENAs emitted from the dayside upper atmosphere of Mars. The energy spectrum is approximated by two-segment power law function joint at the transition energy. The median values of the transition energy and the slopes for the low- and high-energy parts are $E_{0}=607$ $\mathrm{eV}, k_{1}=-2.74$ and $k_{2}=-3.14$, respectively. Compared to the observed charge-exchange HENAs from the flank of Mars and Venus, the interplanetary space and the heliosheath, our results show a lower transition energy and softer spectrum in the low-energy part. These differences are consistent with the spectral features of backscattered HENAs predicted by theory and numerical simulations.

However, the median directional flux of observed HENAs is $1.12 \times 10^{5} \mathrm{~cm}^{-2} \mathrm{~s}^{-1} \mathrm{sr}^{-1}$, is too high for scattered HENAs considering the solar wind proton flux and exospheric densities during the period of observation. The observed spectra also spread more widely than the model expectation for backscattered upstream HENAs. To investigate these problems, we modeled the full HENA production in the dayside plasma environment of Mars, including the upstream and the magnetosheath productions via the charge exchange reaction. The chargeexchanged HENAs contribute to the observation of sunward HENAs by two means: 1) They can penetrate the magnetosphere, precipitate to the exobase and be scattered sunward, contributing to the low-energy part of the measured spectrum. 2) A small fraction of them initially has sunward velocity components, contributing to the high-energy part of the measured spectrum. Our current observations provide the evidence of these sunward-gyrating protons and thus emphasize the kinetic nature of the solar wind interaction of Mars.

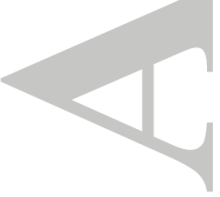




\section{Acknowledgments}

The work of X.-D. W. is supported by the Swedish National Space Agency under the contract 107/12. V. S. was supported by the Russian Science Foundation (RSCF Project 19-1200370). The NPD data used in this work can be obtained at https://data.irf.se/wang2019jgr .

\section{References}

Acuna, M. H. (1998). Magnetic Field and Plasma Observations at Mars: Initial Results of the Mars Global Surveyor Mission. Science, 279(5357), 1676-1680. https://doi.org/10.1126/science.279.5357.1676

Barabash, S., Lundin, R., Andersson, H., Brinkfeldt, K., Grigoriev, A., Gunell, H., et al. (2006). The Analyzer of Space Plasmas and Energetic Atoms (ASPERA-3) for the Mars Express Mission. Space Science Reviews, 126(1-4), 113-164.

Diéval, C., Kallio, E., Barabash, S., Stenberg, G., Nilsson, H., Futaana, Y., et al. (2012). A case study of proton precipitation at Mars: Mars Express observations and hybrid simulations. Journal of Geophysical Research, 117(A6), A06222-A06222. https://doi.org/10.1029/2012JA017537

Diéval, C., Stenberg, G., Nilsson, H., \& Barabash, S. (2013). A statistical study of proton precipitation onto the Martian upper atmosphere: Mars Express observations. $J$. Geophys. Res. Sp. Phys., 118(5), 1972-1983.

Dubinin, E., Fränz, M., Woch, J., Roussos, E., Barabash, S., Lundin, R., et al. (2006). Plasma Morphology at Mars. Aspera-3 Observations. Space Science Reviews, 126(1), 209 238. https://doi.org/10.1007/s11214-006-9039-4

Futaana, Y., Barabash, S., Grigoriev, A., Holmström, M., Kallio, E., Brandt, P. C., Gunell, H., Brinkfeldt, K., Lundin, R., Andersson, H., Yamauchi, M., McKenna-Lawler, S., Winningham, J. D., Frahm, R. A., Sharber, J. R., Scherrer, J. R., Coates, A. J., Linder, D. R., Kataria, D. O., Säles, T., Riihelä, P., Schmidt, W., Koskinen, H., Kozyra, J., Luhmann, J., Roelof, E., Williams, D., Livi, S., Curtis, C. C., Hsieh, K. C., Sandel, B. R., Grande, M., Carter, M., Sauvaud, J.-A., Fedorov, A., Thocaven, J.-J., Orsini, S., Cerulli-Irelli, R., Maggi, M., Wurz, P., Bochsler, P., Galli, A., et al. (2006a). First ENA observations at Mars: ENA emissions from the Martian upper atmosphere. Icarus, 182(2), 424-430. https://doi.org/10.1016/j.icarus.2005.09.019

Futaana, Y., Barabash, S., Grigoriev, A., Holmström, M., Kallio, E., Brandt, P., Gunell, H., Brinkfeldt, K., Lundin, R., Andersson, H., Yamauchi, M., McKenna-Lawler, S., Winningham, D., Frahm, R., Sharber, J. R., Scherrer, J. R., Coates, A., Linder, D. R., Kataria, D. O., Säles, T., Riihelä, P., Schmidt, W., Koskinen, H., Kozyra, J., Luhmann, J., Roelof, E., Williams, D., Livi, S., Curtis, C. C., Hsieh, K. C., Sandel, B. R., Grande, M., Carter, M., Sauvaud, J.-A., Fedorov, A., Thocaven, J.-J., Orsini, S., Cerulli-Irelli, R., Maggi, M., Wurz, P., Bochsler, P., Krupp, N., et al. (2006b). First ENA observations at Mars: Subsolar ENA jet. Icarus, 182(2), 413-423. https://doi.org/10.1016/j.icarus.2005.08.024

Futaana, Y., Chaufray, J. Y., Smith, H., Garnier, P., Lichtenegger, H., Delva, M., et al. (2011). Exospheres and Energetic Neutral Atoms of Mars, Venus and Titan. Space Science Reviews, 162(1), 213-266. https://doi.org/10.1007/s11214-011-9834-4 
Futaana, Y., Barabash, S., Wieser, M., Holmström, M., Lue, C., Wurz, P., et al. (2012). Empirical energy spectra of neutralized solar wind protons from the lunar regolith. Journal of Geophysical Research, 117(E5), E05005-E05005. https://doi.org/10.1029/2011JE004019

Galli, A., Wurz, P., Barabash, S., Grigoriev, A., Gunell, H., Lundin, R., et al. (2006). Energetic Hydrogen and Oxygen Atoms Observed on the Nightside of Mars. Space Science Reviews, 126(1), 267-297.

Galli, A., Wurz, P., Bochsler, P., Barabash, S., Grigoriev, A., Futaana, Y., et al. (2008). First observation of energetic neutral atoms in the Venus environment. Planetary and Space Science, 56(6), 807-811.

Galli, A., Wurz, P., Kallio, E., Ekenbäck, A., Holmström, M., Barabash, S., et al. (2008). Tailward flow of energetic neutral atoms observed at Mars. Journal of Geophysical Research, 113(E12). https://doi.org/10.1029/2008JE003139

Galli, A., Fok, M.-C., Wurz, P., Barabash, S., Grigoriev, A., Futaana, Y., et al. (2008). Tailward flow of energetic neutral atoms observed at Venus. Journal of Geophysical Research, 113(E12), E00B15. https://doi.org/10.1029/2008JE003096

Grigoriev, A. (2007). The Neutral Particle Detector on the Mars and Venus Express missions. Swedish Institute of Space Physics.

Halekas, J. S. (2017). Seasonal variability of the hydrogen exosphere of Mars. Journal of Geophysical Research: Planets, 122(5), 901-911. https://doi.org/10.1002/2017JE005306

Kallio, E., \& Barabash, S. (2000). On the elastic and inelastic collisions between precipitating energetic hydrogen atoms and Martian atmospheric neutrals. Journal of Geophysical Research, 105(A11), 24973-24996. https://doi.org/10.1029/2000JA900077

Kallio, E., Luhmann, J. G., \& Barabash, S. (1997). Charge exchange near Mars: The solar wind absorption and energetic neutral atom production. Journal of Geophysical Research, 102(A10), 22183-22197. https://doi.org/10.1029/97JA01662

Kallio, E., Liu, K., Jarvinen, R., Pohjola, V., \& Janhunen, P. (2010). Oxygen ion escape at Mars in a hybrid model: High energy and low energy ions. Icarus, 206(1), 152-163.

Nilsson, H., Stenberg, G., Futaana, Y., Holmström, M., Barabash, S., Lundin, R., et al. (2012). Ion distributions in the vicinity of Mars: Signatures of heating and acceleration processes. Earth, Planets and Space, 64(2), 135-148. https://doi.org/10.5047/eps.2011.04.011

Ramstad, R., Barabash, S., Futaana, Y., Nilsson, H., Wang, X.-D., \& Holmström, M. (2015). The Martian atmospheric ion escape rate dependence on solar wind and solar EUV conditions: 1. Seven years of Mars Express observations. Journal of Geophysical Research: Planets, 120(7), 1298-1309. https://doi.org/10.1002/2015JE004816

Shematovich, V. I., Bisikalo, D. V., \& Gérard, J. C. (1994). A kinetic model of the formation of the hot oxygen geocorona: 1. Quiet geomagnetic conditions. Journal of Geophysical Research, 99(A12), 23217. https://doi.org/10.1029/94JA01769

Shematovich, V. I., Bisikalo, D. V., Dil’ $\{$ e $\}$ val, C., Barabash, S., Stenberg, G., Nilsson, H., et al. (2011). Proton and hydrogen atom transport in the Martian upper atmosphere with 
an induced magnetic field. Journal of Geophysical Research, 116(A11).

https://doi.org/10.1029/2011JA017007

Wang, X.-D., Barabash, S., Futaana, Y., Grigoriev, A., \& Wurz, P. (2013). Directionality and variability of energetic neutral hydrogen fluxes observed by Mars Express. $J$. Geophys. Res. Sp. Phys., 118(12), 7635-7642. https://doi.org/10.1002/2013JA018876

Wang, X.-D., Alho, M., Jarvinen, R., Kallio, E., Barabash, S., \& Futaana, Y. (2016). Emission of hydrogen energetic neutral atoms from the Martian subsolar magnetosheath. Journal of Geophysical Research: Space Physics, 121(1), 190-204. https://doi.org/10.1002/2015JA021653

Wang, X.-D., Alho, M., Jarvinen, R., Kallio, E., Barabash, S., \& Futaana, Y. (2018). Precipitation of Hydrogen Energetic Neutral Atoms at the Upper Atmosphere of Mars. Journal of Geophysical Research: Space Physics. https://doi.org/10.1029/2018JA025188

Wurz, P., Scheer, J. J., \& Wieser, M. (2006). Particle Scattering off Surfaces: Application in Space Science: E-Journal Surf. Sci. Nanotechnol., 4, 394-400.
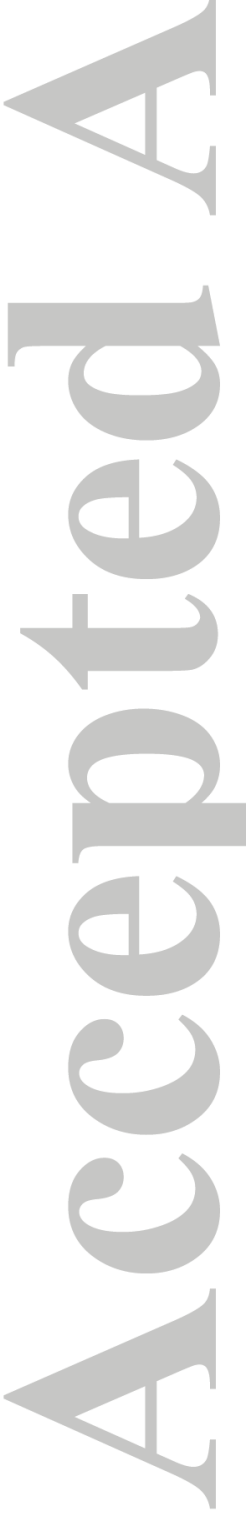